\title{
Impact of air-sea exchange coefficients on the structure and intensity of tropical cyclones
}

\author{
Young Cheol Kwon ${ }^{1}$ and Taekyun Kim ${ }^{2, *}$ \\ ${ }^{1}$ Korea Institute of Atmospheric Prediction Systems, Seoul, Korea \\ ${ }^{2}$ Earth System Research Division, National Institute of Meteorological Sciences, Seogwipo-si, Korea
}

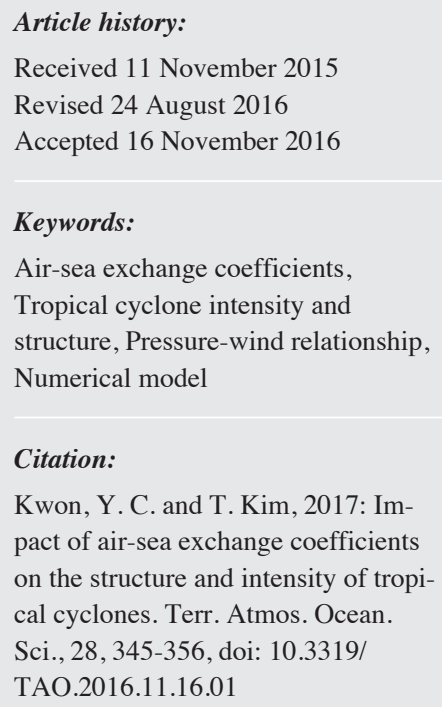

\begin{abstract}
To examine the impact of air-sea exchange coefficients on the structure and intensity of tropical cyclones, sensitivity experiments are performed using the Weather Research and Forecasting (WRF) Model. A typhoon-like idealized vortex embedded in a calm environment is simulated using combinations of three different values of airsea momentum and enthalpy exchange coefficients. In order to investigate the clear roles of each exchange coefficient, the experiments are designed that the two exchange coefficients change independently. For example, while the enthalpy exchange coefficient varies, the momentum exchange coefficient keeps its original value and vice versa. The track and intensity of sensitivity tests are analyzed to check the validity of the experiments. Results show that the track of the idealized vortices under a calm environment remains stationary, while the intensity varies with different air-sea exchange coefficients. The results indicate that changes in storm intensity with different enthalpy exchange coefficients are mainly related to an alteration in the amount of energy input to the storm, whereas the intensity changes with different momentum exchange coefficient values mostly result from angular momentum conservation due to changes in the size of the vortex. There is little change in the net energy input to the storms when the values of the momentum exchange coefficient are changed. Calculations of the volume-integrated angular momentum at the radius of the maximum winds confirm the different intensifying mechanisms found in this study.
\end{abstract}

\section{INTRODUCTION}

Of all severe weather events, tropical cyclones are one of the most destructive meteorological phenomena. The loss of human life and economic assets due to tropical cyclones have increased in recent years because of the increase of human population and development in typhoon-prone coastal areas (Pielke and Landsea 1998). Therefore, an ability to make accurate predictions of the track and intensity of typhoons with an adequate lead time would assist in reducing the damages caused by typhoons. Typhoon track forecast skills have been greatly improved, as shown in a number of studies over several decades (DeMaria et al. 2005; Rogers et al. 2006; Rappaport et al. 2009), mainly due to advancements in the large scale prediction skills of numerical weather prediction models. However, the prediction skills relating to typhoon intensity and structure have not been im-

\footnotetext{
* Corresponding author

E-mail:tkkim79@gmail.com
}

proved as much compared to track skills.

The tracks of tropical storms are mainly governed by large-scale flows, while the intensity and structural changes in storms mostly depend on many factors such as smaller scale convection, multi-scale interactions, and other environmental factors like vertical wind shear and sea surface temperature (SST) (Weidinger et al. 2000). In recent years certain projects have focused on improving the forecast skills of tropical cyclone intensity, such as the Hurricane Forecast Improvement Program (HFIP) made significant progress regarding hurricane intensity and structure forecast (Gall et al. 2013). The accurate prediction of the intensity and structure of tropical storms is also important in predicting storm surge, inland flooding, and other down-stream applications. Storm surge and inland flooding caused by typhoons are two of the most damaging events caused by landfalling storms (Shultz et al. 2005; Peduzzi et al. 2012), and precise prediction of the size and intensity of a typhoon 
is required in order to make an accurate forecast for such events. However, this is impeded by a lack of understanding of the physical and dynamical mechanisms controlling the intensity and size of a tropical storm.

Several environmental factors, such as vertical wind shear and SST, have long been recognized as one of the governing factors in tropical storm intensity (Gray et al. 1997; Landsea et al. 1999; Goldenberg et al. 2001; Bell and Chelliah 2006). High SST and weak vertical wind shear are well known to be favorable conditions for intensifying tropical cyclones and vice versa. In addition to these environmental factors, air-sea exchange of energy in the form of momentum and enthalpy is also known as one of the key processes in controlling the intensity and possibly size of tropical storms (Montgomery et al. 2010; Green and Zhang 2013). Ooyama (1969, hereafter O69) investigated the sensitivity of air-sea exchange coefficient on the intensity of a tropical storm using a numerical model. He found that the intensity is positively proportional to the enthalpy exchange coefficient $\left(\mathrm{C}_{\mathrm{H}}\right)$ but negatively proportional to the momentum exchange coefficient $\left(\mathrm{C}_{\mathrm{D}}\right)$. This result is intuitively correct because $C_{D}$ represents the rate of energy loss of tropical storm while $\mathrm{C}_{\mathrm{H}}$ represents the energy gain. Using axisymmetric balanced model, Emanuel (1995, hereafter E95) also showed that the maximum tangential wind speed is proportional to the square root of $C_{H}$ over $C_{D}$ which is consistent with O69's result. Emanuel also suggested that the ratio of $\mathrm{C}_{\mathrm{H}}$ over $\mathrm{C}_{\mathrm{D}}$ should be larger than 0.75 for a storm to develop stronger than category-3 stage.

Some recent studies on the effects of air-sea exchange coefficients on tropical cyclones have shown somewhat different results compared to O69 and E95. For example, Montgomery et al. (2010) conducted sensitivity of $C_{D}$ to intensity and the size of an idealized tropical storm while keeping $\mathrm{C}_{\mathrm{H}}$ constant. Their results suggested that the idealized storm intensifies with increased $C_{D}$ to a certain critical value and weakened afterward instead of continuing to weaken with increased $C_{D}$. They also concluded that a storm can be a major hurricane with the value of the ratio $C_{H}$ over $C_{D}$ as low as 0.1. Bryan (2012) performed a series of axisymmetric numerical experiments with wide range of $C_{D}, C_{H}$, horizontal and vertical mixing length. His study showed that horizontal mixing length is the most sensitive to tropical storm intensity and structure. Instead of evaluating the impact of $C_{D}$ and $\mathrm{C}_{\mathrm{H}}$ independently, the sensitivity of the $\mathrm{C}_{\mathrm{H}} / \mathrm{C}_{\mathrm{D}}$ ratio to storm size and intensity was examined in his study. Although the intensifying rates differed with the horizontal mixing length, a storm intensifies generally with increased $C_{H} / C_{D}$ ratio. On the other hand, the size of a storm is not affected much by this ratio according to his results. Based on numerical simulations of Hurricane Katrina (2005), Green and Zhang (2013) reported that the intensity is more sensitive to $C_{H}$ changes while $C_{D}$ has more impact on the pressure-wind relationship similar to Bao et al. (2012).
The direct observation of flux values over the ocean in high wind speeds has not been available until recent field experiments, such as CBLAST (Coupled Boundary Layer Air-Sea Transfer) (Black et al. 2007). The general consensus of the $C_{D}$ profile over the ocean is that it increases linearly with wind speeds at a certain point and levels-off over the critical wind speeds. However, the exact starting point of the wind speed level-off is not clear, while several observations suggested it may vary around the $25-30 \mathrm{~m} \mathrm{~s}^{-1}$ range at $\mathrm{h}=10 \mathrm{~m}$ (Powell et al. 2003; Donelan et al. 2004; Bell et al. 2012). On the other hand, the $C_{H}$ is believed to remain at the same value regardless of wind speed changes (Black et al. 2007; Bell et al. 2012), different from the conventional idea of linearly increasing with wind speed. However, caution is needed because the spread of the exchange coefficients over the ocean is quite large and there could be significant errors in observations (e.g., Montgomery et al. 2010).

In this study, we are trying to see the impacts of the air-sea exchange coefficients with a slightly different approach compared to most of the previous studies. The $C_{D}$ and $\mathrm{C}_{\mathrm{H}}$ are not set constant but rather change with the wind speed, and they are allowed to vary with stability, as in the real atmosphere. In addition, the momentum and thermal roughness length $\left(\mathrm{Z}_{0}\right.$ and $\left.\mathrm{Z}_{\mathrm{T}}\right)$ are modified consistently to change the exchange coefficients. Because $C_{D}$ and $C_{H}$ are functions of $Z_{0}$ and $Z_{T}$, the change in $C_{D}$ and $C_{H}$ without modifying $\mathrm{Z}_{0}$ and $\mathrm{Z}_{\mathrm{T}}$ could cause inconsistencies, especially in the physics schemes that use the roughness length like a boundary layer scheme. Finally, we keep the $\mathrm{C}_{\mathrm{H}}$ value when $C_{D}$ varies, and keep the $C_{D}$ value when $C_{H}$ varies in order to see the impacts of each coefficient clearly. Because both $C_{D}$ and $C_{H}$ are related via $Z_{0}$, the process of modifying one coefficient without changing the other one requires some algebraic manipulations. This process will be described in the next section. In this context, the impact of the air-sea exchange coefficients on the hurricane intensity and structure of a typhoon-like vortex is investigated in an idealized vortex using a Weather Research and Forecasting (WRF) model. Three different profiles of $C_{D}$ and $C_{H}$ are designed for the sensitivity experiments, and the idealized vortex is embedded in a calm environment. The simulation results are then analyzed to examine how varying exchange coefficients alter the intensity and structure of a tropical cyclone.

The experimental design and method for modifying $C_{D}$ and $\mathrm{C}_{\mathrm{H}}$ are described in section 2 . The results are presented in section 3, where the basic fields and storm structures are shown in relation to the sensitivity results. In addition, two different mechanisms of storm intensity changes in relation to $C_{D}$ and $C_{H}$ are explained and the related structural impact of the storm on these coefficients is described. Finally, section 4 presents a summary and the conclusion.

\section{METHODOLOGY}




\subsection{Experimental Design}

The WRF model version 3.6.1 and the idealized vortex option provided by the WRF model (Rotunno and Emanuel 1987) are used for the sensitivity experiments in this study. The horizontal resolution of the experiments is $9 \mathrm{~km}$ with $601 \times 601$ grid points, and 41 vertical levels with a modeltop height of $25 \mathrm{~km}$. The surface of the entire model domain is covered by ocean with a constant SST of $28^{\circ} \mathrm{C}$ and a constant Coriolis parameter of $5 \times 10^{-5} \mathrm{~s}^{-1}\left(20^{\circ} \mathrm{N}\right)$. The idealized vortex is set to be axisymmetric with a maximum wind speed of $15 \mathrm{~m} \mathrm{~s}^{-1}$ and an initial radius of $82.5 \mathrm{~km}$. The size of the vortex, the radius at which the typhoon wind component becomes zero, is set to $412 \mathrm{~km}$. Figure 1 shows the plane view of the sea level pressure and wind fields and the vertical cross-section of the tangential wind of the initial condition used in this study.

The lateral boundary is set to a periodic condition in both the east-west and north-south directions. The MoninObukhov similarity method is used for calculation of surface fluxes, the Yon-Sei University (YSU) scheme is used for boundary layer parameterization, and the Rapid Radiative Transfer Mode (RRTM-G)/Dudhia schemes are used for short/long wave radiation. Convection is parameterized using the Kain-Fritch scheme, while the grid scale moisture process is represented by the WRF Single Moment-6 (WSM6) scheme. The model simulations are made to $\mathrm{t}=$ $120 \mathrm{~h}$ with an hourly output.

\subsection{Air-Sea Exchange Coefficients}

The bulk surface fluxes of momentum, sensible heat, and latent heat can be written as Eqs. (1), (2), and (3), respectively. $\tau=-\rho C_{D}(U)^{2}$

$S H=\rho c_{p} C_{H} U \Delta \theta$

$L H=\rho L_{v} C_{Q} U \Delta q$

where $\rho$ is the density of air, $U$ is the wind speed at a reference level (usually $\mathrm{h}=10 \mathrm{~m}$ ), $\Delta \theta$ and $\Delta q$ are the differences in potential temperature and the mixing ratio at the surface and at the reference level, respectively. $C_{D}, C_{H}$, and $C_{Q}$ are the bulk exchange coefficients for momentum, heat, and moisture, respectively. As in most surface schemes, the exchange coefficients for heat and moisture are set the same in our study.

The bulk exchange coefficients can be expressed as Eqs. (4) and (5) using the Monin-Obukhov similarity theory.

$$
\begin{aligned}
& C_{D}=\frac{k}{\ln \left(\frac{z_{1}}{z_{0}}\right)-\psi_{m}\left(\frac{z_{1}}{L}\right)} \times \frac{k}{\ln \left(\frac{z_{1}}{z_{0}}\right)-\psi_{m}\left(\frac{z_{1}}{L}\right)} \\
& C_{H}=\frac{k}{\ln \left(\frac{z_{1}}{z_{0}}\right)-\psi_{m}\left(\frac{z_{1}}{L}\right)} \times \frac{k}{\ln \left(\frac{z_{1}}{z_{T}}\right)-\psi_{h}\left(\frac{z_{1}}{L}\right)}
\end{aligned}
$$

where $k$ is the von Kármán constant. $z_{0}$ and $z_{T}$ are the momentum and the thermal roughness length, respectively. $\psi_{m}$ and $\psi_{h}$ are the universal function for momentum and enthalpy, which is a function of the atmospheric stability. These universal functions have values of zero under neutral conditions.

In order to perform the sensitivity experiments of the
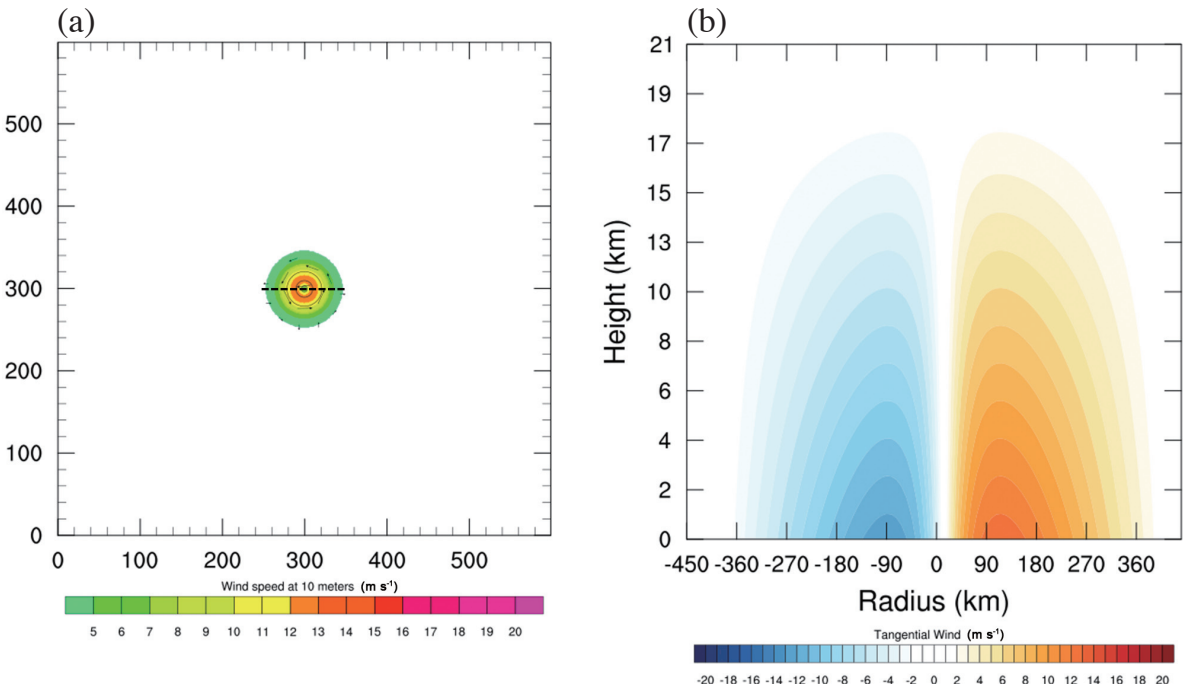

Fig. 1. (a) $10 \mathrm{~m}$ wind fields (arrow and shaded with color) and (b) vertical cross-section of tangential wind along the dashed line in (a) at the initial time. The minimum value of sea level pressure is set to $1021 \mathrm{hPa}$. (Color online only) 
momentum $\left(\mathrm{C}_{\mathrm{D}}\right)$ and enthalpy $\left(\mathrm{C}_{\mathrm{H}}\right)$ exchange coefficients, systematic modifications of these coefficients are made as shown in Fig. 2. Three $C_{D}$ profiles are designed. The first profile increases linearly with wind speed, the second levels off at a wind speed of approximately $20 \mathrm{~m} \mathrm{~s}^{-1}$, and the third decreases at a wind speed over $20 \mathrm{~m} \mathrm{~s}^{-1}$ (Fig. 2a). In addition, three $\mathrm{C}_{\mathrm{H}}$ profiles are also designed. The first increases slightly, the second is steady, and the third decreases slightly (as seen in Fig. 2b).

According to Eqs. (4) and (5), $C_{D}$ is solely a function of the momentum roughness length, while $\mathrm{C}_{\mathrm{H}}$ depends on both momentum and the thermal roughness length under neutral conditions. Therefore, given the $\mathrm{C}_{\mathrm{D}}$ and $\mathrm{C}_{\mathrm{H}}$ profiles with a neutral assumption, $z_{0}$ and $z_{T}$ can be obtained by simple algebraic manipulations because there are two unknowns $\left(z_{0}\right.$ and $z_{T}$ ) and two equations [Eqs. (4) and (5)]. The final exchange coefficients are obtained using the calculated $z_{0}, z_{T}$ and actual universal function terms. Figure 3 shows the momentum and thermal roughness length obtained using this method. Five experiments are conducted in this study using various air-sea enthalpy and momentum exchange coefficients, and these are summarized in Table 1.

\section{RESULTS}

\subsection{Basic Fields}

Figure 4 shows the time series of the intensity of the $\mathrm{C}_{\mathrm{D}}$ sensitivity experiments (Fig. $4 \mathrm{a}$ ) and the $\mathrm{C}_{\mathrm{H}}$ experiments (Fig. 4b). As expected, when the $C_{D}$ values become smaller and the $\mathrm{C}_{\mathrm{H}}$ values become larger, the storm intensity becomes stronger in terms of both the central pressure and the maximum wind speed at $\mathrm{h}=10 \mathrm{~m}$, and vice versa. The tracks are not shown because the vortex remains at the same position during whole forecast periods. One noteworthy feature in the intensity plots is that there is a greater variation in the values of maximum wind speed in the $C_{D}$ experiments than in the $\mathrm{C}_{\mathrm{H}}$ experiments. However, there is an almost identical spread of the central pressure values in both experiments.
In this study, the storm intensity in each experiment does not reach the steady state. One of the main reasons some idealized vortices do not reach might be the physics suites settings, especially the radiation and boundary layer mixing schemes (Hakim 2011; Bao et al. 2012). In addition, the ever-changing characteristics of convections in most threedimensional numerical weather prediction simulations are usually known to cause asymmetries of tropical cyclones. The asymmetries caused by moist convections tend to produce some degree of high or medium frequency oscillations of storm intensity. Of course the careful settings let idealized vortices remain in a steady state but this is not a critical factor in our opinion. Therefore, the conclusion made in this study may not be invalid because the storm does not reach the steady state.

The mean sea level pressure and $10 \mathrm{~m}$ height wind speeds averaged between $t=84 \mathrm{~h}$ and $\mathrm{t}=96 \mathrm{~h}$ are shown in Fig. 5. The central pressure and maximum wind speed values in the plane view are consistent with the intensity features in Fig. 4. Larger $C_{D}$ and smaller $C_{H}$ produce weaker storms and smaller $\mathrm{C}_{\mathrm{D}}$ and larger $\mathrm{C}_{\mathrm{H}}$ produce strong storms. Mean flows do not exist and the Coriolis parameter is set to constant, so the structures of the simulated vortices remain mostly axisymmetric. Based on time evolutions of intensity, track, and horizontal structures, the simulations conducted in this study contain no unforeseen problems in relation to the idealized vortices and lateral boundary conditions used in this study.

\subsection{Storm Structure}

A model-simulated relationship between the central pressure and maximum wind speeds is one of the key metrics for evaluating a model's performance regarding typhoon structures (Bao et al. 2012; Green and Zhang 2013). Storm intensity, storm size, and other structure-related information can be deduced by analyzing the pressure-wind relationship. The size of the storm can be defined several ways, such as (a)

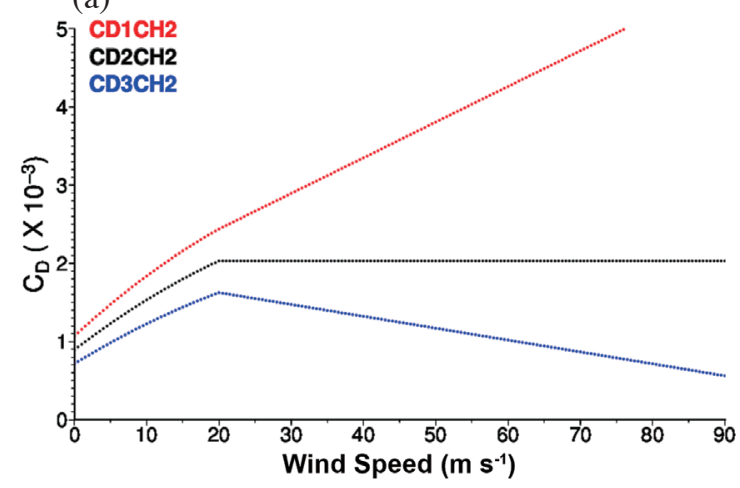

(b)

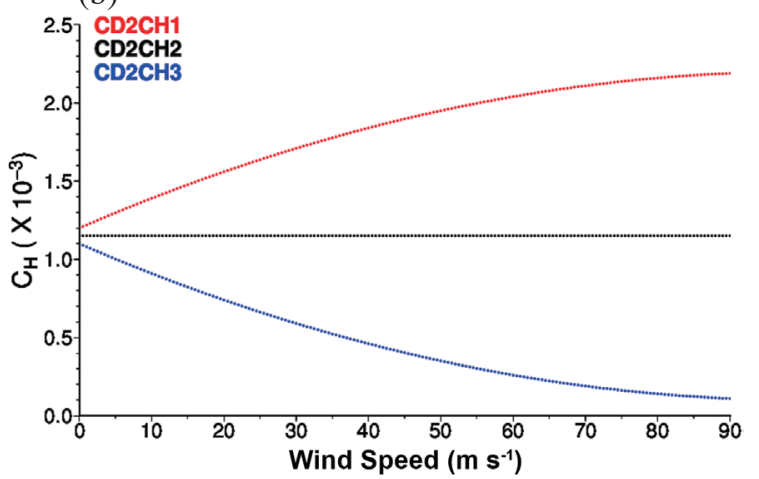

Fig. 2. (a) Plots of the exchange coefficients for momentum flux, $C_{D}$, as functions of $10 \mathrm{~m}$ wind speed used in this study; (b) as in (a) but the exchange coefficients of enthalpy, $\mathrm{C}_{\mathrm{H}}$, (cf. Table 1). (Color online only) 
(a)

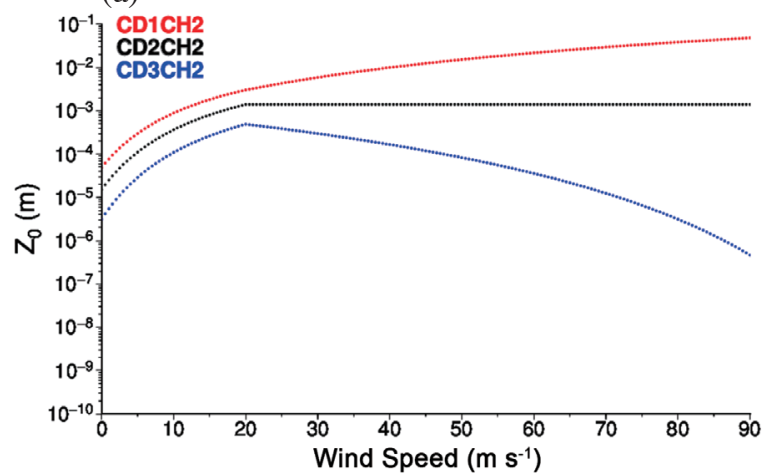

(b)

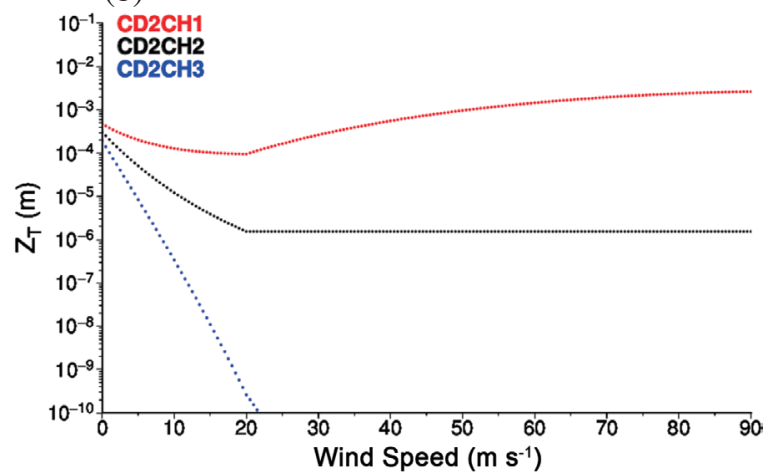

Fig. 3. (a) Plots of momentum roughness length, $\mathrm{Z}_{0}$, as functions of $10 \mathrm{~m}$ wind speed obtained in this study; (b) as in (a), but the thermal roughness length, $\mathrm{Z}_{\mathrm{T}}$. (Color online only)

Table 1. List of experiments.

\begin{tabular}{cc}
\hline Experiment Name & Description \\
\hline $\mathrm{CD} 1 \mathrm{CH} 2$ & Linearly increasing $\mathrm{C}_{\mathrm{D}}$ and Steady $\mathrm{C}_{\mathrm{H}}$ \\
$\mathrm{CD} 3 \mathrm{CH} 2$ & Decreasing $\mathrm{C}_{\mathrm{D}}$ at a wind speed over $20 \mathrm{~m} \mathrm{~s}^{-1}$ and Steady $\mathrm{C}_{\mathrm{H}}$ \\
$\mathrm{CD} 2 \mathrm{CH} 2$ & Leveling off $\mathrm{C}_{\mathrm{D}}$ at a wind speed over $20 \mathrm{~m} \mathrm{~s}^{-1}$ and Steady $\mathrm{C}_{\mathrm{H}}$ \\
$\mathrm{CD} 2 \mathrm{CH} 1$ & Slightly increasing $\mathrm{C}_{\mathrm{H}}$ and leveling off $\mathrm{C}_{\mathrm{D}}$ at a wind speed over $20 \mathrm{~m} \mathrm{~s}^{-1}$ \\
$\mathrm{CD} 2 \mathrm{CH} 3$ & Slightly decreasing $\mathrm{C}_{\mathrm{H}}$ and leveling off $\mathrm{C}_{\mathrm{D}}$ at a wind speed over $20 \mathrm{~m} \mathrm{~s}^{-1}$ \\
\hline
\end{tabular}

(a)
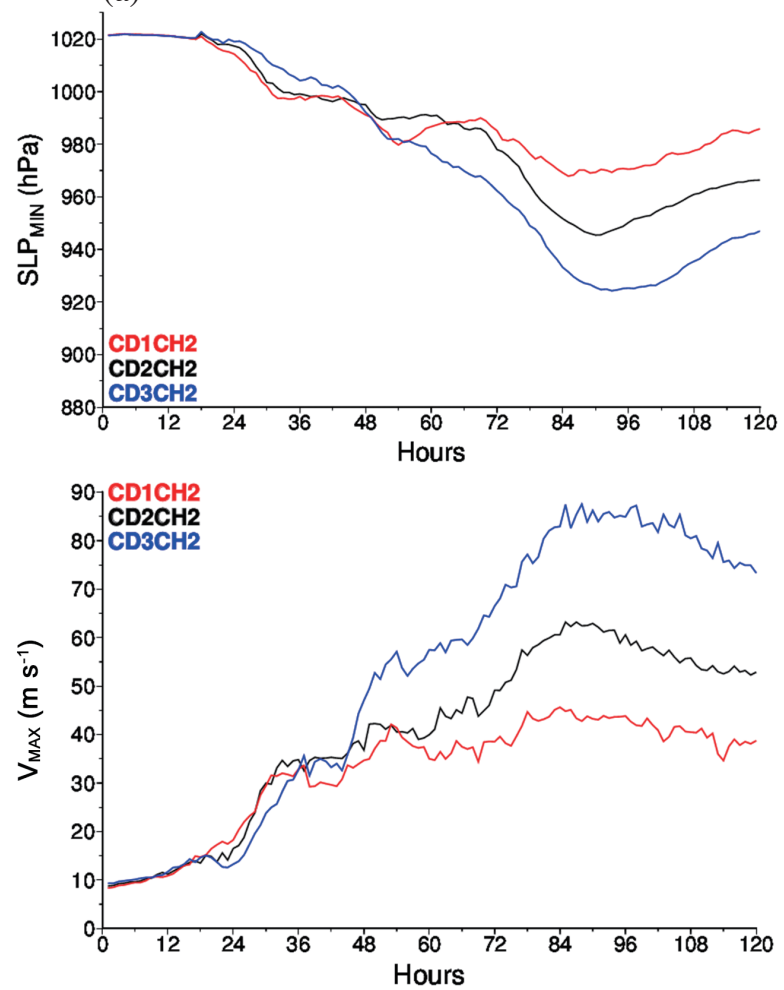

(b)
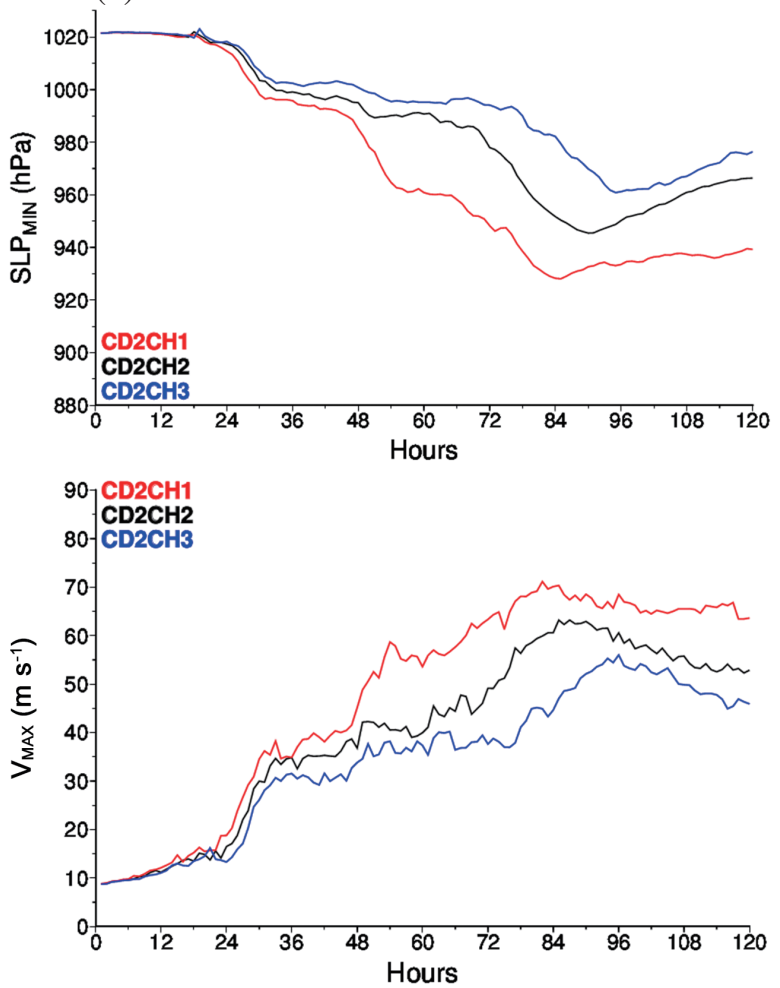

Fig. 4. (a) Time series of minimum sea level and maximum $10 \mathrm{~m}$ wind speed of the $\mathrm{C}_{\mathrm{D}}$ sensitivity experiments: $\mathrm{CD} 1 \mathrm{CH} 2$ (red), $\mathrm{CD} 2 \mathrm{CH} 2$ (black), and $\mathrm{CD} 3 \mathrm{CH} 2$ (blue) for a 120-h period; (b) as in (a) but of the $\mathrm{C}_{\mathrm{H}}$ sensitivity experiments: CD2CH1 (red), $\mathrm{CD} 2 \mathrm{CH} 2$ (black), and CD2CH3 (blue). (Color online only) 

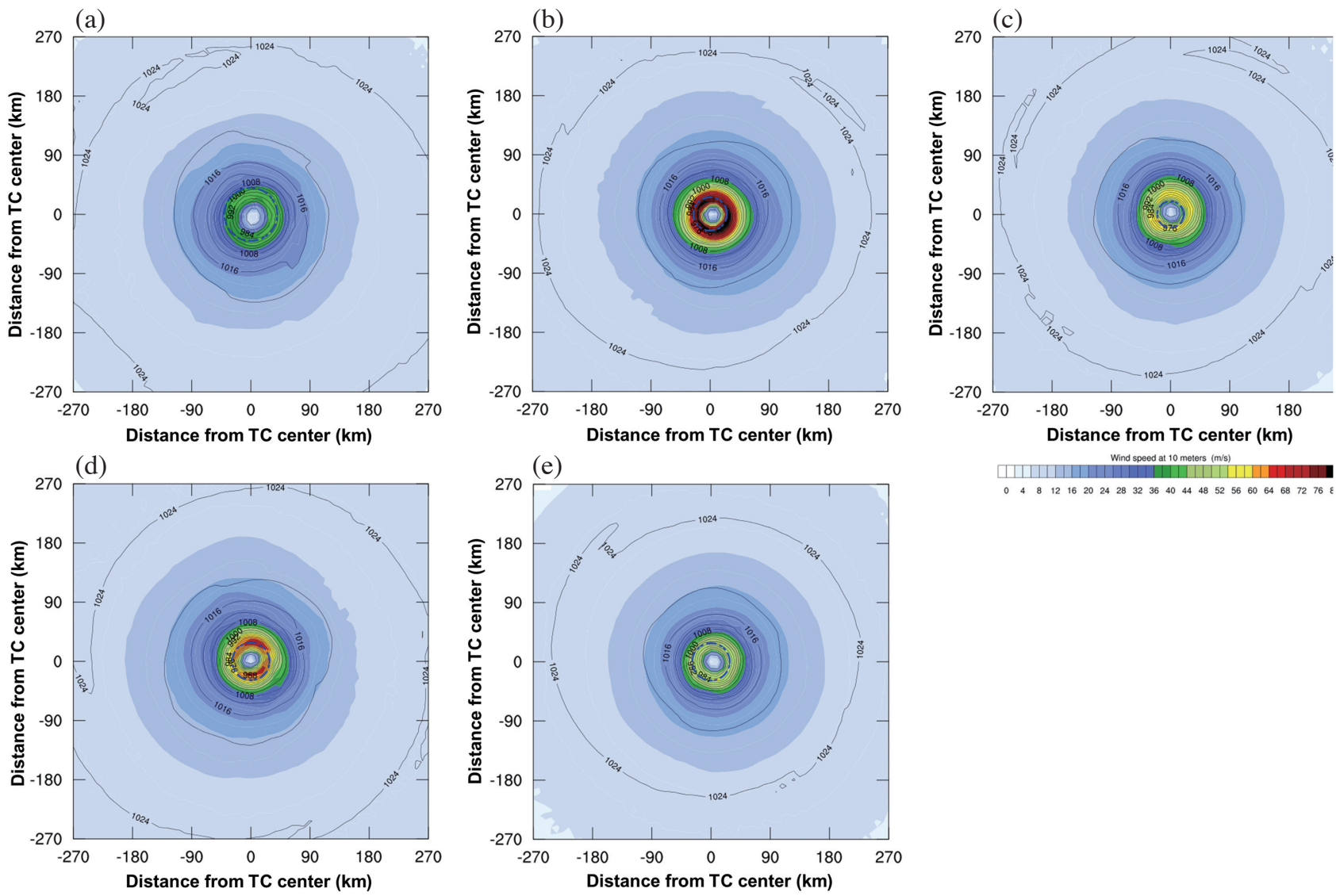

Fig. 5. Plan view of the simulated sea level pressure (contours every $4 \mathrm{hPa}$ ) and $10 \mathrm{~m}$ wind speed (color-shaded) averaged between 84 and $96 \mathrm{~h}$ for experiments: (a) $\mathrm{CD} 1 \mathrm{CH} 2$, (b) $\mathrm{CD} 3 \mathrm{CH} 2$, (c) $\mathrm{CD} 2 \mathrm{CH} 2$, (d) $\mathrm{CD} 2 \mathrm{CH} 1$, and (e) $\mathrm{CD} 2 \mathrm{CH} 3$. The blue-dashed line indicates the radius of the maximum winds. (Color online only)

the outer most closed isobar, radius of 34-kt wind, or radius of maximum winds (RMW). Although most of the academic papers use the storm size as 34-kt wind radius, the size is defined as RMW in this study. One of the main reasons for using RMW as the storm size is that the pressure-wind relationship is between the maximum wind speed and central pressure of a tropical storm. So using RMW is the straightforward way to explain the size and pressure-wind relationship. According to our analysis, RMW and 34-kt wind radius do not have much differences, which confirms the rational for using RMW. The pressure-wind relationships obtained from the $\mathrm{C}_{\mathrm{D}}$ and $\mathrm{C}_{\mathrm{H}}$ sensitivity experiments are shown in Fig. 6, where dots with different colors represent the various experiments using different air-sea exchange coefficients. The dots are plotted using the central pressure and maximum wind speed values from an hourly output of $120 \mathrm{~h}$ simulations in each experiment. The lower-right corner of the graph represents a weak storm, where low wind speeds exist with high central pressure, while the upper-left corner of the graph represents a strong storm with high wind speeds and low central pressure.

The pressure-wind relationship lines of the $C_{D}$ and $C_{H}$ sensitivity results show completely different behaviors in terms of slope. The slope of the pressure-wind lines changes when a different value of $C_{D}$ is used, whereas the slope of the lines does not change with changes in the value of $\mathrm{C}_{\mathrm{H}}$. The slope change in the pressure-wind lines indicates that the maximum wind speeds are different for the same central pressure value. For example, when the central pressure value is $960 \mathrm{hPa}$, the maximum wind speed varies between 40,50 , and $60 \mathrm{~m} \mathrm{~s}^{-1}$ with a different value of $C_{D}$ (Fig. 6a). These maximum wind changes at the same central pressure value are possible because the maximum wind speed of a typhoon is proportional to the radial pressure gradient, not to the central pressure value itself. In order to create higher wind speeds at a given central pressure, the size of a storm needs to be smaller, so the radial pressure gradient becomes bigger. In this respect, the pressure-wind relationship in the $C_{D}$ experiments suggests a change in the size of the storm. However, changes in the value of $\mathrm{C}_{\mathrm{H}}$ have no effect on the slope of the pressure-wind relation lines, although the intensity becomes stronger with higher $\mathrm{C}_{\mathrm{H}}$ values (Fig. 6b).

Hovmöller diagrams of azimuthally averaged tangential wind speed are used to confirm the different storm size sensitivity shown by the pressure-wind relationship, as presented in Fig. 7. From the picture, it can be seen that when 

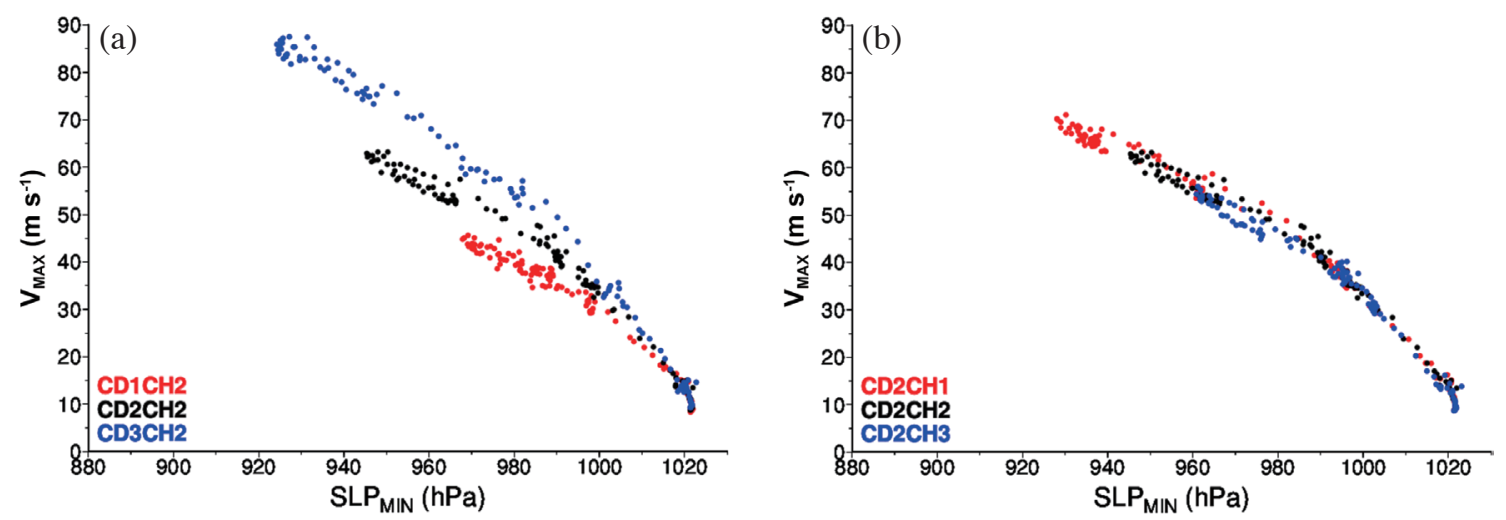

Fig. 6. (a) Scatterplot of minimum sea level vs maximum $10 \mathrm{~m}$ wind speed of the $\mathrm{C}_{\mathrm{D}}$ sensitivity experiments: $\mathrm{CD} 1 \mathrm{CH} 2$ (red), $\mathrm{CD} 2 \mathrm{CH} 2$ (black), and $\mathrm{CD} 3 \mathrm{CH} 2$ (blue) for a 120-h period; (b) as in (a) but of $\mathrm{C}_{\mathrm{H}}$ sensitivity experiments: $\mathrm{CD} 2 \mathrm{CH} 1$ (red), $\mathrm{CD} 2 \mathrm{CH} 2$ (black), and $\mathrm{CD} 2 \mathrm{CH} 3$ (blue). (Color online only)

(a)

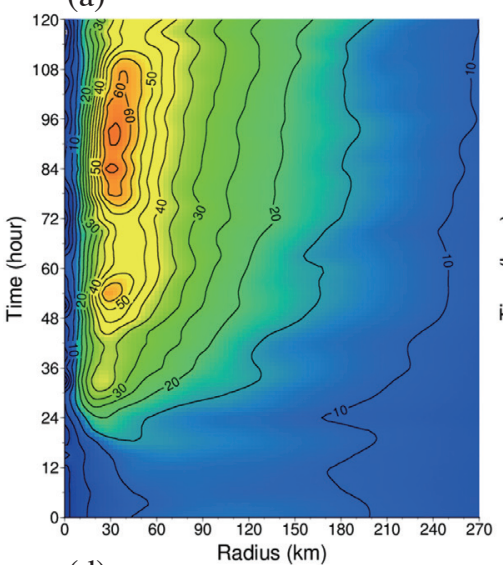

(d)

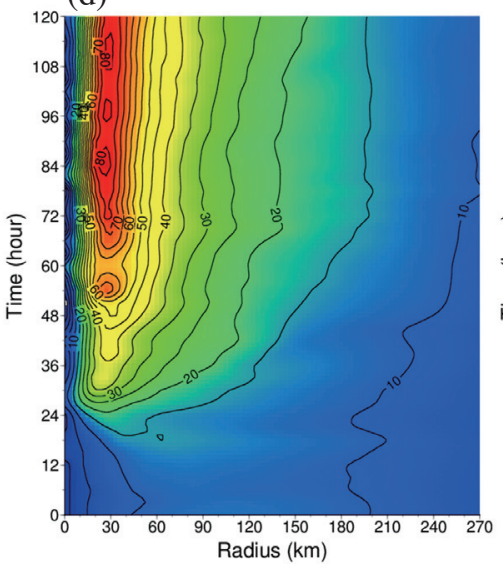

(b)

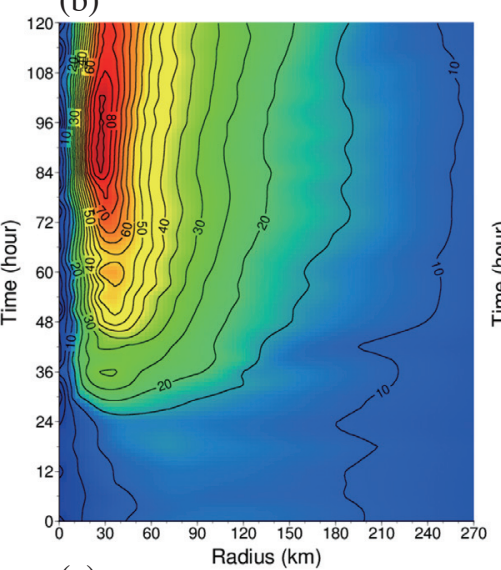

(e) (c)

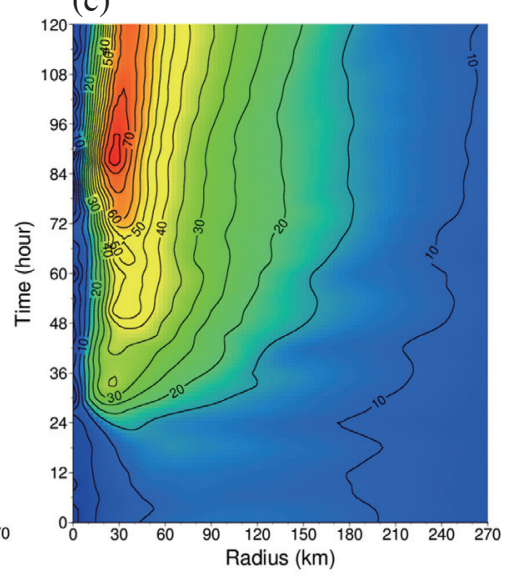

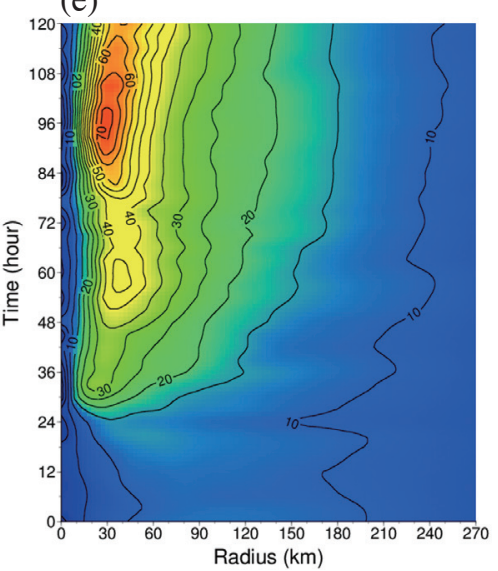

Fig. 7. Hovmöller plots of azimuthally averaged tangential wind-speed at $1 \mathrm{~km}$ height for a 120 -h period: (a) $\mathrm{CD} 1 \mathrm{CH} 2$, (b) $\mathrm{CD} 3 \mathrm{CH} 2$, (c) $\mathrm{CD} 2 \mathrm{CH} 2$, (d) $\mathrm{CD} 2 \mathrm{CH} 1$, and (e) $\mathrm{CD} 2 \mathrm{CH} 3$. (Color online only)

the simulated storm has larger values of $\mathrm{C}_{\mathrm{D}}(\mathrm{CD} 1 \mathrm{CH} 2)$, the RMW expands with time. However, when the storm has smaller values of $\mathrm{C}_{\mathrm{D}}(\mathrm{CD} 3 \mathrm{CH} 2)$, the $\mathrm{RMW}$ contracts its size with the simulation time, and this is most notable between $\mathrm{t}=60 \mathrm{~h}$ and $\mathrm{t}=108 \mathrm{~h}$. In contrast, the storm sizes in the $\mathrm{C}_{\mathrm{H}}$ experiments appear to maintain the same radii of
$30-35 \mathrm{~km}$. The radial profiles of tangential wind and radial wind speed averaged over $\mathrm{t}=84 \mathrm{~h}$ to $\mathrm{t}=96 \mathrm{~h}$ at $\mathrm{h}=1$ $\mathrm{km}$ are shown in Fig. 8. The tangential wind speeds located around the typhoon core become stronger with decreasing values of $C_{D}$, and the RMW also become smaller at the same time (Fig. 8a). However, the radius of the maximum wind 
is maintained almost same distance in the $\mathrm{C}_{\mathrm{H}}$ sensitivity experiments, although larger values of $\mathrm{C}_{\mathrm{H}}$ produce a stronger storm (Fig. 8b).

The radial wind profiles provide an interesting insight into the different storm size sensitivities of the two experiments. While the magnitude of the radial wind speed remains almost same, the locations of the maximum radial wind speeds are different in the $C_{D}$ sensitivity tests. When the value of $C_{D}$ becomes smaller, the location of the maximum radial wind becomes closer to the storm center (Fig. 8c). However, this type of location change is not seen in $\mathrm{C}_{\mathrm{H}}$ experiments, but instead the magnitude of the radial wind speed increases with an increase in the value of $\mathrm{C}_{\mathrm{H}}$ (Fig. 8d). Our analysis indicates that the radially inward flows penetrate closer to a storm's center due to a reduction in surface angular momentum loss. The difference in the radial inflow penetration depth, which is caused by $C_{D}$, is one of the reasons responsible for changes in the size of a storm.

It is known that when the size of a typhoon shrinks, the tangential wind speeds of a storm increase due to angular momentum conservation. It is, therefore, possible that a part of the intensity changes in the $C_{D}$ experiments could be caused by the contraction of a storm in addition to the reduction in the energy sink due to the reduction in surface drag. Conversely, there is no change in the storm size in the
$\mathrm{C}_{\mathrm{H}}$ experiments, and therefore the intensity differences in the $\mathrm{C}_{\mathrm{H}}$ experiments could have been mostly caused by the increased energy source due to enhanced surface enthalpy fluxes.

The angular momentum at the RMW of the vortices simulated by the increased $(\mathrm{CD} 1 \mathrm{CH} 2)$ and decreased $(\mathrm{CD} 3 \mathrm{CH} 2)$ $C_{D}$ experiments at $t=96 \mathrm{~h}$ are calculated to examine what proportion of the intensity changes are due to vortex contraction. The $10 \mathrm{~m}$ maximum wind speed is $40.6 \mathrm{~m} \mathrm{~s}^{-1}$ and the RMW is $49.5 \mathrm{~km}$ for the $\mathrm{CD} 1 \mathrm{CH} 2$ vortex, while those of the $\mathrm{CD} 3 \mathrm{CH} 2$ vortex are $75.3 \mathrm{~m} \mathrm{~s}^{-1}$ and $26.8 \mathrm{~km}$, respectively. Therefore, the angular momentum at the RMW of $\mathrm{CD} 1 \mathrm{CH} 2$ is $2.01 \times 10^{6} \mathrm{~m}^{2} \mathrm{~s}^{-1}$, and that of $\mathrm{CD} 3 \mathrm{CH} 2$ is $2.02 \times 10^{6} \mathrm{~m}^{2} \mathrm{~s}^{-1}$. Thus, most of the changes in typhoon intensity changes in $C_{D}$ are caused by vortex contraction rather than the reduction in surface drag that occurs when smaller values of $C_{D}$ are used in this study.

In contrast, the angular momentum calculation in the $\mathrm{C}_{\mathrm{H}}$ experiments confirms that they have very different values for the angular momentum at the RMW with various values of $\mathrm{C}_{\mathrm{H}}, 2.19 \times 10^{6} \mathrm{~m}^{2} \mathrm{~s}^{-1}\left(57.7 \mathrm{~m} \mathrm{~s}^{-1} \times 37.95 \mathrm{~km}\right)$ and $1.59 \times 10^{6} \mathrm{~m}^{2} \mathrm{~s}^{-1}\left(46.9 \mathrm{~m} \mathrm{~s}^{-1} \times 33.94 \mathrm{~km}\right)$ for $\mathrm{CD} 2 \mathrm{CH} 1$ and $\mathrm{CD} 2 \mathrm{CH} 3$, respectively. This result indicates that the intensity changes in the $\mathrm{C}_{\mathrm{H}}$ experiments are mainly caused by changes in energy input to the vortex. The $10 \mathrm{~m}$ maximum
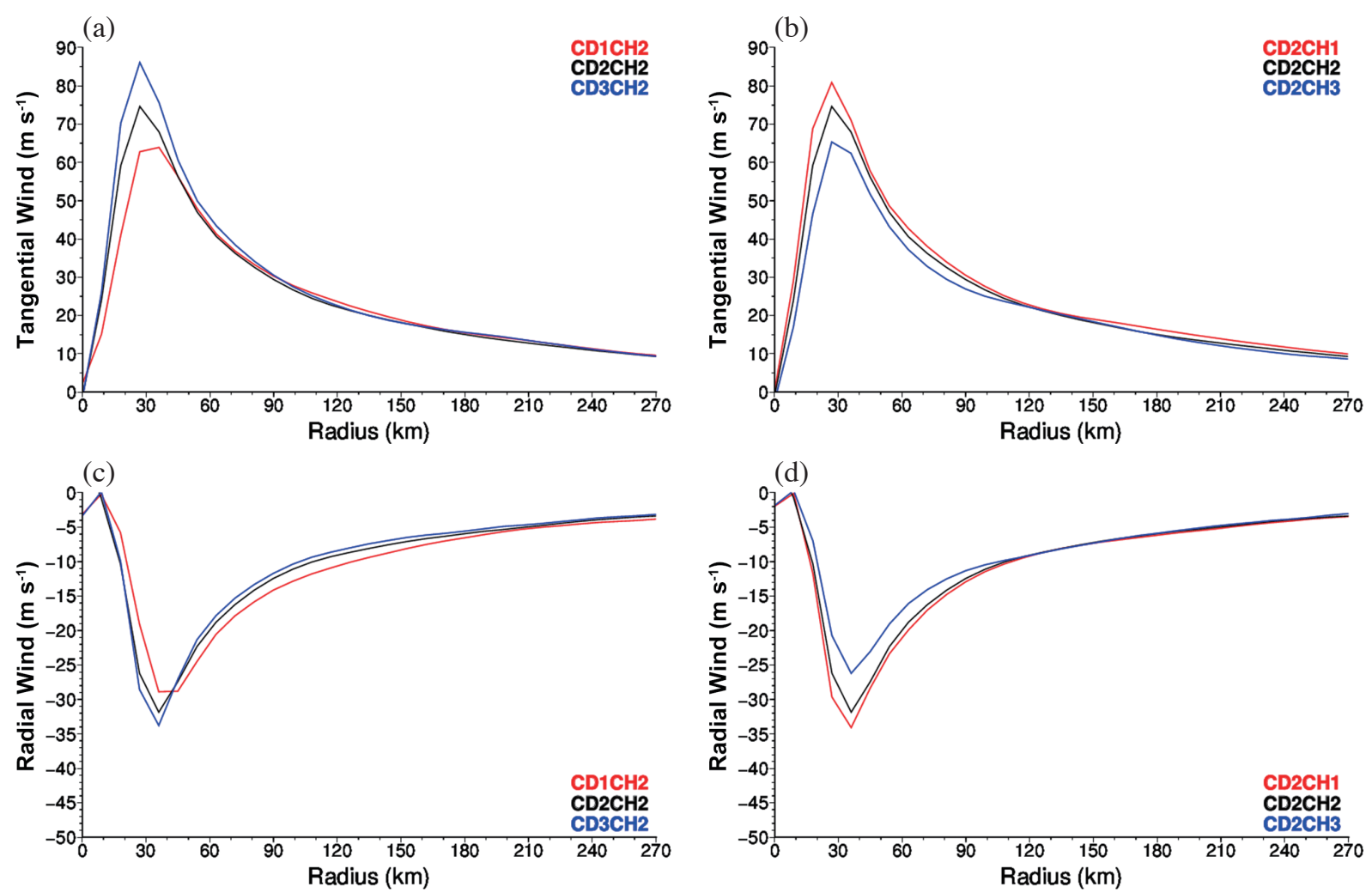

Fig. 8. Radial profiles of azimuthally mean tangential (a) (b) and radial (c) (d) wind-speeds inside the inflow layer (at $1 \mathrm{~km}$ height), averaged between 84 and 96 h of the $\mathrm{C}_{\mathrm{D}}$ sensitivity experiments (a) (c): $\mathrm{CD} 1 \mathrm{CH} 2$ (red), $\mathrm{CD} 2 \mathrm{CH} 2$ (black), and $\mathrm{CD} 3 \mathrm{CH} 2$ (blue); and of the $\mathrm{C}_{\mathrm{H}}$ sensitivity experiments (b) (c): $\mathrm{CD} 2 \mathrm{CH} 1$ (red), $\mathrm{CD} 2 \mathrm{CH} 2$ (black), and $\mathrm{CD} 2 \mathrm{CH} 3$ (blue). (Color online only) 
wind speed with increased $\mathrm{C}_{\mathrm{H}}$ values $\left(57.7 \mathrm{~m} \mathrm{~s}^{-1}\right)$ is stronger than that of decreased $\mathrm{C}_{\mathrm{H}}$ values $\left(46.9 \mathrm{~m} \mathrm{~s}^{-1}\right)$, while the RMW with increased $C_{H}$ values $(37.95 \mathrm{~km}$ ) remains similar to that of the decreased $\mathrm{C}_{\mathrm{H}}$ values $(33.94 \mathrm{~km})$. In the aspect of the angular momentum conservation, the positive intensity change due to $\mathrm{C}_{\mathrm{H}}$ would be considered an enhancement of the net energy to the storm system.

According to the angular momentum calculations of the experiments in this study, changes in surface drag modify the storm intensity mostly through changing the size of the storm, whereas the enthalpy exchange coefficient changes the storm intensity via the net energy input to the system. The angular momentum of the vortices in the $C_{D}$ and $\mathrm{C}_{\mathrm{H}}$ sensitivity experiments are calculated and plotted as time series (Fig. 9) in order to check how the total angular momentum of the storm system alters with changes in the air-sea exchange coefficients. The angular momentum is volume integrated from the surface to model top $(\mathrm{h}=21$ $\mathrm{km}$ ) and from the center of a storm to $\mathrm{r}=270 \mathrm{~km}$. Consistent with the previous argument, the volume integrated angular momentum of the $\mathrm{C}_{\mathrm{H}}$ experiments is more sensitive than that of the $C_{D}$ experiments. While the change in $C_{D}$ has shown little sensitivity on the volume integrated angular momentum, especially in later time of simulation periods (after $t$ $=60 \mathrm{~h}$ ), the decrease in $\mathrm{C}_{\mathrm{H}}$ shows the systematic reduction in the volume integrated angular momentum. This indicates that the loss and gain in angular momentum are balanced in the case of $C_{D}$ experiments, while the gain in angular momentum is greater than the loss in angular momentum with bigger $\mathrm{C}_{\mathrm{H}}$. In order to confirm this, the net advection of angular momentum along the border of $r=270 \mathrm{~km}$ cylinder is calculated. The change of the angular momentum inside the cylinder is caused by horizontal advection along the edge of the cylinder, pressure torque, and surface friction. According to the previous angular momentum budget study, the angular momentum changes in a tropical cyclone are mainly caused by horizontal advection and surface friction (Kang and Cheong 2012). The loss of angular momentum due to surface friction and gain/loss due to horizontal advection of the sensitivity experiments are calculated, and their normalized values to the control experiment are plotted in the form of time series (Fig. 10). The red lines denote the horizontal advection and the blue lines are the surface friction effect. As can be seen in Fig. 10, the loss in surface friction does not change much with changes in both $C_{D}$ and $C_{H}$ although the magnitude of friction is slightly more sensitive to $C_{D}$. On the other hand, the horizontal advection terms are much more sensitive to the change in $\mathrm{C}_{\mathrm{H}}$ (Figs. 10c and d) than that in $\mathrm{C}_{\mathrm{D}}$ (Figs. 10a and b). As a result, the sum of these two terms (Fig. 11) showed clear differences, which are less sensitive for $C_{D}$ (Fig. 11a) and more sensitive for $C_{H}$ (Fig. 11b). This result is consistent with Fig. 9, which shows similar volume integrated angular momentum values in the $C_{D}$ experiments. These results indicate that the modification of $\mathrm{C}_{\mathrm{H}}$ modulates the angular momentum of the storm system whereas that of $\mathrm{C}_{\mathrm{D}}$ does not impact much.

\section{DISCUSSION AND CONCLUSION}

The accurate predictions of intensity and structure of typhoons are important to avoid the damage they cause. However, the mechanisms involved in the physical and dynamical processes of typhoon intensity changes and the ensuing structural evolutions are not well understood. Several possible reasons for the limited improvement in intensity and structural forecast skills could be a lack of understanding of the internal dynamics of typhoon vortices, the complicated interaction between large scale and small-scale vortices, and improper representations of physical processes in relation to the scarcity of available observation data on tropical storms.

In this study, the impacts of air-sea momentum and
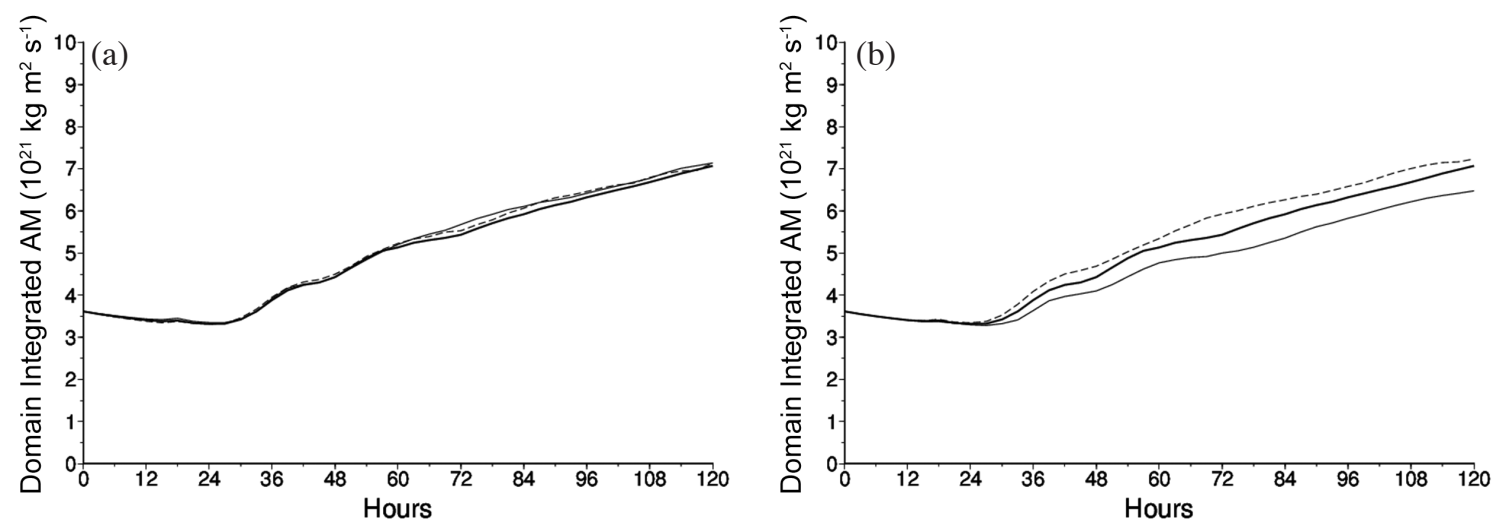

Fig. 9. (a) Time series of volume-integrated angular momentum of the $\mathrm{C}_{\mathrm{D}}$ sensitivity experiments: $\mathrm{CD} 1 \mathrm{CH} 2$ (dashed line), $\mathrm{CD} 2 \mathrm{CH} 2$ (thick line), and $\mathrm{CD} 3 \mathrm{CH} 2$ (thin line), for a 120-h period; (b) as in (a) but of the $\mathrm{C}_{\mathrm{H}}$ sensitivity experiments: $\mathrm{CD} 2 \mathrm{CH} 1$ (dashed line), $\mathrm{CD} 2 \mathrm{CH} 2$ (thick line), and $\mathrm{CD} 2 \mathrm{CH} 3$ (thin line). The angular momentum in the plots is volume integrated from surface to model top $(\sim 21 \mathrm{~km})$, and from the center of the storm to $\mathrm{r}=270 \mathrm{~km}$. 

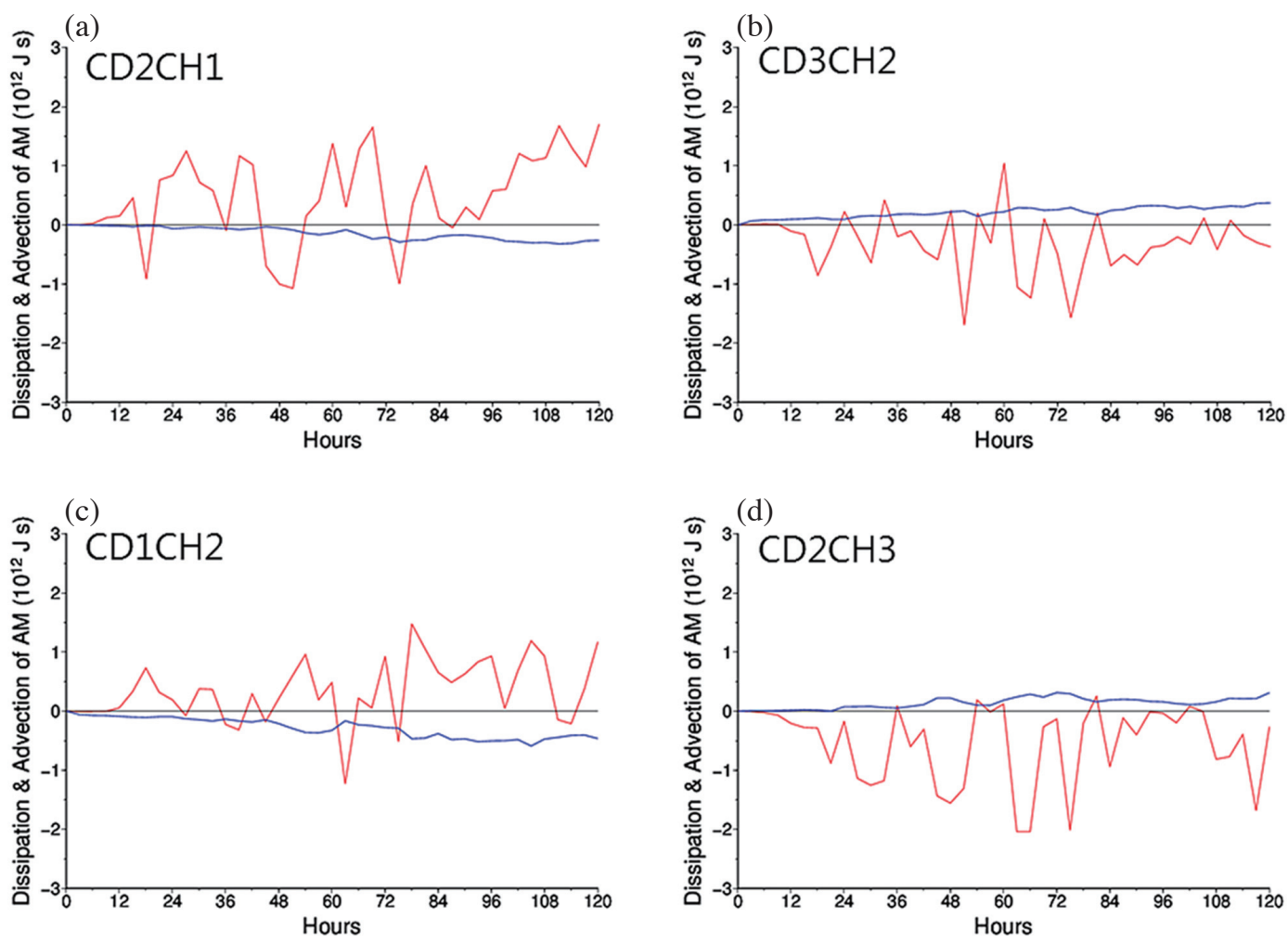

Fig. 10. The time series of the angular momentum loss due to surface friction (blue lines) and gain/loss due to horizontal advection (red lines) normalized to the control experiment (CD2CH2). Upper panels are for $\mathrm{CD}$ experiments (a) (b) and lower panels are for $\mathrm{CH}$ experiments (c) (d), respectively. (Color online only)
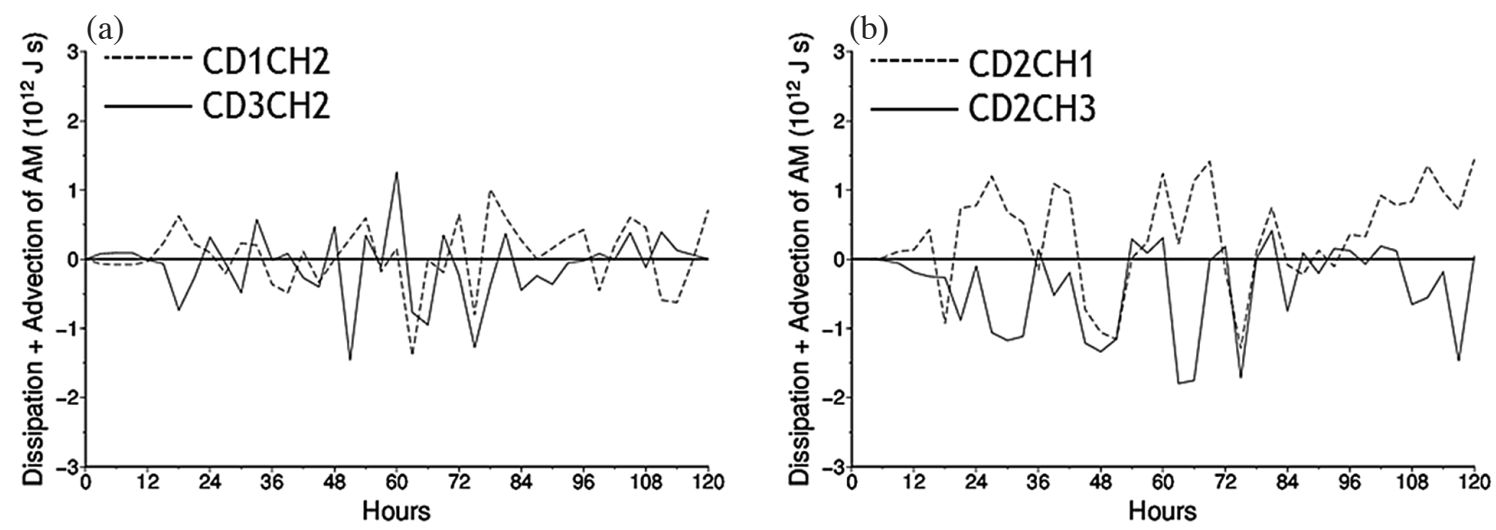

Fig. 11. Summation of the effect of the surface friction and horizontal advection for (a) $\mathrm{CD}$ and (b) $\mathrm{CH}$ experiments. The zero line indicates the value of the control experiment. Note that the net effects are quite smaller in $\mathrm{CD}$ experiment than in $\mathrm{CH}$ experiments.

enthalpy exchange processes, regarded as the first order energy sink and source of a typhoon, are investigated using numerical sensitivity experiments. An idealized typhoonlike vortex in a calm environment is simulated using the WRF model with the different air-sea exchange coefficients designed in this study. The intensity and tracks of the sensitivity experiments produce results similar to those in previous studies. The increase of the $\mathrm{C}_{\mathrm{H}}$ or the decrease of the $\mathrm{C}_{\mathrm{D}}$ intensifies the storm intensity, both in terms of central pressure and maximum wind speeds (e.g., Ooyama 1969; Emanuel 1995).

The structural evolution of the simulated storms shows distinctly different features between the $C_{D}$ and $C_{H}$ sensitivity tests. The pressure-wind relationships and Hovmöller diagrams of azimuthally averaged tangential wind speed show that the size of the storm measured by the RMW behaves differently in the two sensitivity experiments. While the size of the storm mostly remains the same with changes 
in $\mathrm{C}_{\mathrm{H}}$, the $\mathrm{C}_{\mathrm{D}}$ experiments produce different size storms. The use of smaller values of $C_{D}$ produces smaller size storms and larger $C_{D}$ values produce larger storms. The analysis conducted in this study indicates that reduced surface drag with smaller values of $C_{D}$ causes a deeper penetration of the radial inflow to the storm's center, which appears to be mainly responsible for the change in size. In contrast, $\mathrm{C}_{\mathrm{H}}$ sensitivity tests show no change in the radial flow penetration and of the storm's size. There are some similarities and differences between our results and the results from the previous studies. The pressure-wind relationship depends on mostly $C_{D}$ not $C_{H}$ confirmed in this study is the same as most of the previous research (Bao et al. 2012; Green and Zhang 2013). However, the size of tropical storm increases with larger $C_{D}$ is opposite to the results of Montgomery et al. (2010) and Green and Zhang (2013). Although the detailed analysis for this discrepancy will be one of the topics for a future study, we want to point out some differences in experimental settings in our work to that of others. First, the air-sea exchange coefficients in our study are designed to have dependency on the wind speed different to the usage of the constant wind speed as in Montgomery et al. (2010). Second, the coefficients of this study also include the effect of stability functions different from two previous studies. Finally, the inter-connection of $C_{D}$ and $C_{H}$ via $Z_{0}$ is removed with a simple algebraic manipulation of the equations, so the experiments performed in this study are clearly able to examine the sensitivity of each coefficient separately. Therefore, we believe that these differences might contribute the evolution of storm size with varying $C_{D}$.

Further analysis reveals that the storm intensity changes caused by changing $C_{D}$ and $C_{H}$ values do not result from the same processes. When the enthalpy exchange coefficient is increased, the net energy input to the storm increases and the storm therefore intensifies. In contrast, the change in intensity due to changes in $C_{D}$ values does not result from an alteration in the net energy input to the storm but rather caused by angular momentum conservation due to the change in the storm size. Angular momentum values at the RMW in the different $C_{D}$ simulations are almost the same, which is proof that a change in storm intensity with various values of $C_{D}$ is mostly caused by size changes and not total energy changes. However, angular momentums at the RMW do not remain the same in weak and strong storms in the $\mathrm{C}_{\mathrm{H}}$ experiments. Angular momentum is a conserved variable, and therefore the different angular momentum of two vortices means that there is a net change in the storm energy.

The results of this study indicate that the size and structure of storms are more sensitive to the air-sea momentum exchange coefficient than the enthalpy exchange coefficient. This is one of the new findings in our study and further analysis is in progress to confirm the robustness of our conclusion. Although a change in the enthalpy exchange coefficient alters the net energy of the storm, it does not change the storm structure mainly in terms of the size. In contrast, a change in the value of the momentum exchange coefficient modifies the structures and the storm intensity. In fact, the change in intensity due to the momentum exchange coefficient is mostly due to the size and angular momentum conservation not to the energy changes in the storm.

In future studies we plan to conduct further analyses using the results from this current study to investigate the detailed processes involved in alterations of the structure of the typhoon-like vortex in relation to changes in surface drag. In addition, the sea-state dependent air-sea exchange processes will be examined using an atmosphere-wave coupled system, instead of using simple atmospheric wind-speed dependent air-sea exchange parameterization. We are expecting that the atmosphere-wave coupled system will enable us to gain a better understanding of the relationship between the evolution of tropical storms and sea state changes.

Acknowledgements We thank the three anonymous reviewers for their constructive comments and suggestions to improve this manuscript. This work has been carried out through the R\&D project on the development of global numerical weather prediction systems of Korea Institute of Atmospheric Prediction Systems (KIAPS) funded by Korea Meteorological Administration (KMA). Also, T. Kim was supported by the project "Research and Development for KMA Weather, Climate, and Earth system Services" funded by National Institute of Meteorological Sciences (NIMS).

\section{REFERENCES}

Bao, J. W., S. G. Gopalakrishnan, S. A. Michelson, F. D. Marks, and M. T. Montgomery, 2012: Impact of physics representations in the HWRFX on simulated hurricane structure and pressure-wind relationships. Mon. Weather Rev., 140, 3278-3299, doi: 10.1175/MWRD-11-00332.1. [Link]

Bell, G. D. and M. Chelliah, 2006: Leading tropical modes associated with interannual and multidecadal fluctuations in north Atlantic hurricane activity. J. Climate, 19, 590-612, doi: 10.1175/JCLI3659.1. [Link]

Bell, M., M. Montgomery, and K. Emanuel, 2012: Air-sea enthalpy and momentum exchange at major hurricane wind speeds observed during CBLAST. J.Atmos. Sci., 69, 3197-3222, doi: 10.1175/JAS-D-11-0276.1. [Link]

Black, P. G., E. D'Asaro, W. M. Drennan, J. R. French, P. P. Niiler, T. B. Sanford, E. J. Terrill, E. J. Walsh, and J. A. Zhang, 2007: Air-sea exchange in hurricanes: Synthesis of observations from the Coupled Boundary Layer Air-Sea Transfer experiment. Bull. Amer. Meteorol. Soc., 88, 357-374, doi: 10.1175/BAMS-88-3357. [Link]

Bryan, G. H., 2012: Effects of surface exchange coefficients and turbulence length scales on the intensity and 
structure of numerically simulated hurricanes. Mon. Weather Rev., 140, 1125-1143, doi: 10.1175/MWRD-11-00231.1. [Link]

DeMaria, M., M. Mainelli, L. K. Shay, J. A. Knaff, and J. Kaplan, 2005: Further improvements to the Statistical Hurricane Intensity Prediction Scheme (SHIPS). Weather Forecast., 20, 531-543, doi: 10.1175/ WAF862.1. [Link]

Donelan, M. A., B. K. Haus, N. Reul, W. J. Plant, M. Stianssnie, H. C. Graber, O. B. Brown, and E. S. Saltzman, 2004: On the limiting aerodynamic roughness of the ocean in very strong winds. Geophys. Res. Lett., 31, L18306, doi: 10.1029/2004g1019460. [Link]

Emanuel, K. A., 1995: Sensitivity of tropical cyclones to surface exchange coefficients and a revised steady-state model incorporating eye dynamics. J. Atmos. Sci., 52, 3969-3976, doi: 10.1175/1520-0469(1995)052<3969:S OTCTS $>2.0 . C O ; 2$. [Link]

Gall, R., J. Franklin, F. Marks, E. N. Rappaport, and F. Toepfer, 2013: The hurricane forecast improvement project. Bull. Amer. Meteorol. Soc., 94, 329-343, doi: 10.1175/BAMS-D-12-00071.1. [Link]

Goldenberg, S. B., C. W. Landsea, A. M. Mestas-Nuñez, and W. M. Gray, 2001: The recent increase in Atlantic hurricane activity: Causes and implications. Science, 293, 474-479, doi: 10.1126/science.1060040. [Link]

Gray, W. M., J. D. Sheaffer, and C. W. Landsea, 1997: Climate trends associated with multidecadal variability of Atlantic hurricane activity. In: Diaz, H. F. and R. S. Pulwarty (Eds.), Hurricanes: Climate and Socioeconomic Impacts, Springer, Berlin, Heidelberg, 15-53, doi: 10.1007/978-3-642-60672-4_2. [Link]

Green, B. W. and F. Zhang, 2013: Impacts of air-sea flux parameterizations on the intensity and structure of tropical cyclones. Mon. Weather Rev., 141, 2308-2324, doi: 10.1175/MWR-D-12-00274.1. [Link]

Hakim, G. J., 2011: The mean state of axisymmetric hurricanes in statistical equilibrium. J. Atmos. Sci., 68, 1364-1376, doi: 10.1175/2010JAS3644.1. [Link]

Kang, H.-G. and H.-B. Cheong, 2012: Study on the Angular Momentum of Axisymmetric Tropical Cyclone in the Developing Stage. Atmosphere, 23, 1-11, doi: 10.14191/atmos.2013.23.1.001. [Link]

Landsea, C. W., R. A. Pielke Jr., A. M. Mestas-Nuñez, and J. A. Knaff, 1999: Atlantic basin hurricanes: Indices of climatic changes. Clim. Change, 42, 89-129, doi: 10.1023/A:1005416332322. [Link]
Montgomery, M. T., R. K. Smith, and S. V. Nguyen, 2010: Sensitivity of tropical-cyclone models to the surface drag coefficient. Q. J. R. Meteorol. Soc., 136, 19451953, doi: 10.1002/qj.702. [Link]

Ooyama, K., 1969: Numerical simulation of the life cycle of tropical cyclones. J. Atmos. Sci., 26, 3-40, doi: 10 $.1175 / 1520-0469(1969) 026<0003$ :NSOTLC $>2.0 . C O$; 2. [Link]

Peduzzi, P., B. Chatenoux, H. Dao, A. De Bono, C. Herold, J. Kossin, F. Mouton, and O. Nordbeck, 2012: Global trends in tropical cyclone risk. Nat. Clim. Change, 2, 289-294, doi: 10.1038/nclimate1410. [Link]

Pielke, R. A. and C. W. Landsea, 1998: Normalized hurricane damages in the United States: 1925-95. Weather Forecast., 13, 621-631, doi: 10.1175/1520-0434(1998) 013<0621:NHDITU>2.0.CO;2. [Link]

Powell, M. D., P. J. Vickery, and T. A. Reinhold, 2003: Reduced drag coefficient for high wind speeds in tropical cyclones. Nature, 422, 279-283, doi: 10.1038/nature01481. [Link]

Rappaport, E. N., J. L. Franklin, L. A. Avila, S. R. Baig, J. L. Beven II, E. S. Blake, C. A. Burr, J. G. Jng, C. A. Juckins, R. D. Knabb, C. W. Landsea, M. Mainelli, M. Mayfield, C. L. McAdie, R. J. Pasch, C. Sisko, S. R. Stewart, and A. N. Tribble, 2009: Advances and challenges at the national hurricane center. Weather Forecast., 24, 395419, doi: 10.1175/2008WAF2222128.1. [Link]

Rogers, R., S. Aberson, M. Black, P. Black, J. Cione, P. Dodge, J. Duanion, J. Gamache, J. Kaplan, M. Powell, N. Shay, N. Surgi, and E. Uhlhorn, 2006: The intensity forecasting experiment: A NOAA multiyear field program for improving tropical cyclone intensity forecasts. Bull. Amer. Meteorol. Soc., 87, 1523-1537, doi: 10.1175/BAMS-87-11-1523. [Link]

Rotunno, R. and K. A. Emanuel, 1987: An air-sea interaction theory for tropical cyclones. Part II: Evolutionary study using a nonhydrostatic axisymmetric numerical model. J. Atmos. Sci., 44, 542-561, doi: 10.1175/15200469(1987)044<0542:AAITFT>2.0.CO;2. [Link]

Shultz, J. M., J. Russell, and Z. Espinel, 2005: Epidemiology of tropical cyclones: The dynamics of aisaster, disease, and development. Epidemiol. Rev., 27, 21-35, doi: 10.1093/epirev/mxi011. [Link]

Weidinger, T., J. Pinto, and L. Horváth, 2000: Effects of uncertainties in universal functions, roughness length, and displacement height on the calculation of surface layer fluxes. Meteorol.Z., 9, 139-154. 